\title{
Video Article \\ Ex Vivo Method for Assessing the Mouse Reproductive Tract Spontaneous Motility and a MATLAB-based Uterus Motion Tracking Algorithm for Data Analysis
}

\author{
Kaley L. Liang ${ }^{* 1}$, Julia O. Bursova* ${ }^{1}$, Frank Lam ${ }^{1}$, Xingjuan Chen ${ }^{1}$, Alexander G. Obukhov ${ }^{1}$ \\ ${ }^{1}$ The Department of Anatomy, Cell Biology \& Physiology, Indiana University School of Medicine \\ *These authors contributed equally
}

Correspondence to: Alexander G. Obukhov at aobukhov@iu.edu

URL: https://www.jove.com/video/59848

DOI: doi: $10.3791 / 59848$

Keywords: Biology, Issue 151, uterus, spontaneous uterine motility, epinephrine, reproductive tract, mice, ex vivo model

Date Published: 9/1/2019

Citation: Liang, K.L., Bursova, J.O., Lam, F., Chen, X., Obukhov, A.G. Ex Vivo Method for Assessing the Mouse Reproductive Tract Spontaneous Motility and a MATLAB-based Uterus Motion Tracking Algorithm for Data Analysis. J. Vis. Exp. (151), e59848, doi:10.3791/59848 (2019).

\section{Abstract}

Dysmenorrhea, or painful cramping, is the most common symptom associated with menses in females and its severity can hinder women's everyday lives. Here, we present an easy and inexpensive method that would be instrumental for testing new drugs decreasing uterine contractility. This method utilizes the unique ability of the entire mouse reproductive tract to exhibit spontaneous motility when maintained ex vivo in a Petri dish containing oxygenated Krebs buffer. This spontaneous motility resembles the wave-like myometrial activity of the human uterus, referred to as endometrial waves. To demonstrate the effectiveness of the method, we employed a well-known uterine relaxant drug epinephrine. We demonstrate that the spontaneous motility of the entire mouse reproductive tract can be quickly and reversibly inhibited by $1 \mu \mathrm{M}$ epinephrine in this Petri dish model. Documenting the changes of uterine motility can be easily done using an ordinary smart phone or a sophisticated digital camera. We developed a MATLAB-based algorithm allowing motion tracking to quantify spontaneous uterine motility changes by measuring the rate of uterine horn movements. A major advantage of this ex vivo approach is that the reproductive tract remains intact throughout the entire experiment, preserving all intrinsic intrauterine cellular interactions. The major limitation of this approach is that up to $10-20 \%$ of uteri may exhibit no spontaneous motility. Thus far, this is the first quantitative ex vivo method for assessing spontaneous uterine motility in a Petri dish model.

\section{Video Link}

The video component of this article can be found at https://www.jove.com/video/59848/

\section{Introduction}

As a major female organ, the uterus is crucial for reproduction and essential for the nourishment of the fetus ${ }^{1}$. The uterus consists of three layers: the perimetrium, myometrium and endometrium. The myometrium is the major contractile layer of the uterus and plays a key role in fetus delivery. The endometrium is the innermost layer lining the uterine cavity and is essential for embryo implantation. In non-pregnant females of reproductive age, the endometrial layer is shed monthly at the beginning of the menstrual cycle. The myometrium aids in this shedding process by maintaining the spontaneous myometrial contractions needed for clearing the necrotic endometrial tissue from the uterus ${ }^{1}$.

Unfortunately, increased myometrial contractility can result in negative side effects such as dysmenorrhea, or painful menstrual cramps. This is especially seen in young females and nulliparous women ${ }^{2}$. However, dysmenorrhea is different for every woman and depends on the strength of their myometrial contractions; stronger contractions are often associated with the sensation of severe cramping ${ }^{3}$. Myometrial contractility can be visualized using uterine ultrasound and is often recognized as endometrial waves. Enhanced release of prostaglandins during menstruation ${ }^{4}$ in a uterus undergoing endometrial sloughing is believed to contribute to increased myometrial hypercontractility, resulting in ischemia and hypoxia of the uterine muscle and thus increased pain ${ }^{3}$.

Severe dysmenorrhea can hinder the day-to-day activity of some women and 3 to $33 \%$ of women have very severe pain, which could cause a woman to be bedridden for 1 to 3 days each menstrual cycle ${ }^{5}$. Dysmenorrhea is the leading cause of gynecological morbidity in women of reproductive age regardless of age, nationality, and economic status ${ }^{5}$. The estimated prevalence of dysmenorrhea is both high and variable, ranging from $45 \%$ to $93 \%$ in women of reproductive age $\mathrm{e}^{5}$. Dysmenorrhea-associated pain has an effect on the daily life of women and may result in poor academic performance in adolescents, lower quality of sleep, restriction of daily activities, and mood changes ${ }^{5}$.

Many women who experience severe dysmenorrhea resort to over-the-counter medications to relieve their pain. Such over-the-counter medications contain cyclooxygenase (COX) inhibitors which prevent the formation of prostaglandins ${ }^{6}$. However, COX inhibitors are associated with adverse cardiovascular events, and about $18 \%$ of women with dysmenorrhea are unresponsive to these inhibitors ${ }^{7}$. Therefore, there is a 
need for new medications to reduce menstrual cramps. Since over contractility of the uterus contributes to the pathogenesis of dysmenorrhea, one possible strategy may be the usage of uterine relaxants.

It is beneficial to quantify the effects of potential relaxant drugs in a model of naturally occurring spontaneous myometrial wave-like contractions. However, thus far, no efficient ex vivo method for testing muscle-relaxing drugs in the intact uterus has been described. Currently, isometric tension measurements are used to evaluate relaxant drug effects. During such measurements, a uterine muscle strip is maintained at a constant length under preload in a tissue bath while the force of uterine muscle contractions is recorded before and after oxytocin stimulation in the presence or absence of a relaxant drug. Although this approach is very useful, it requires expensive equipment. Furthermore, isometric contractions do not resemble the spontaneous myometrial wave-like contractions that naturally occur in the intact uterus. Uniquely, the uterine myometrial waves in rodents can be visualized as uterine horn motility when the entire reproductive tract (ovaries, oviducts, uterus, and vagina) is maintained in a buffer solution. Here, we present an ex vivo method for monitoring the spontaneous motility of the intact mouse uterus placed in a Petri dish containing oxygenated Krebs buffer. We also describe a motility quantification algorithm utilizing the MATLAB motion tracker. This novel approach provides an easy and less expensive alternative to test the relaxant potential of naturally occurring remedies and synthetic compounds.

\section{Protocol}

All procedures with animals have been approved by the Institutional Animal Care and Use Committee at the Indiana University School of Medicine (Indianapolis, IN). 2-5 month-old F2-129S-C57BL/6 sexually-mature female mice were used in the study.

CAUTION: Ensure safety by wearing a lab coat, mask, and gloves when working with animals and biohazardous materials.

\section{Solution Preparation}

1. Prepare the Krebs Buffer, which contains: $130 \mathrm{mM} \mathrm{NaCl}, 5 \mathrm{mM} \mathrm{KCl}, 2 \mathrm{mM} \mathrm{CaCl}_{2}, 1.2 \mathrm{mM} \mathrm{NaH}_{2} \mathrm{PO}_{4}, 0.56 \mathrm{mM} \mathrm{MgCl}_{2}, 25 \mathrm{mM} \mathrm{NaHCO}_{3}$, and $5 \mathrm{mM}$ glucose, $\mathrm{pH}$ 7.4. Continuously oxygenate the Krebs buffer with a mixture of compressed gases containing $5 \% \mathrm{CO}_{2}$ and $95 \% \mathrm{O}_{2}$ while maintaining the buffer temperature at $37^{\circ} \mathrm{C}$ using a circulating water bath.

2. Prepare Dulbecco's Phosphate-Buffered Saline (DPBS), which contains: $2.68 \mathrm{mM} \mathrm{KCl}, 1.47 \mathrm{mM} \mathrm{KH}_{2} \mathrm{PO}_{4}, 136.89 \mathrm{mM} \mathrm{NaCl}$, and $8.1 \mathrm{mM}$ $\mathrm{Na}_{2} \mathrm{HPO}_{4}, \mathrm{pH} 7.4$.

\section{Animal Preparation}

1. Anesthetize mice using inhalation of isoflurane (3\%) with waste gas scavenging. Ensure adequate anesthesia by assessing withdrawal reflexes. Pinch the rear toe to affirm no movements are elicited, indicating loss of reflex responses. After the deep anesthesia is achieved, euthanize the animal by decapitation.

NOTE: Isoflurane may cause smooth muscle dilation. Therefore, the reproductive tract preparation should be extensively washed and incubated in the Krebs buffer for at least 15-30 min before beginning the ex vivo experiments. Isoflurane may cause irritation and discomfort when it is in contact with the skin, so proceed with caution.

2. Place the body on a large weigh boat lined with a paper towel.

NOTE: Pregnant female laboratory personnel should not be involved in experiments with isoflurane because it can decrease fetal weight, decrease fetal skeletal ossification, and increase the risk of spontaneous abortion ${ }^{8,9} \cdot \mathrm{CO}_{2}$ inhalation can be used as a substitute to euthanize mice.

\section{Determination of Estrous Cycle Stage}

1. With small forceps, lift the clitoris to gain access to the vaginal ostium and slowly insert a micropipette tip containing $10 \mu \mathrm{L}$ of DPBS into the vagina.

1. Ensure the micropipette tip is inserted through the ostium at an angle of $10-30^{\circ}$ to avoid puncturing the vaginal wall. The liquid should still be visible in the tip after insertion. If the liquid is not visible, the tip was inserted too far into the vagina, and the paracervical area of the vagina might have been perforated.

2. Lightly pull down on the vaginal ostium muscles with the tip of the micropipette to allow air to exit from the vagina.

2. Slowly flush the vaginal cavity by pipetting up and down 2-3 times with $10 \mu \mathrm{L}$ of DPBS and place the drawn cell suspension on a glass slide.

3. Use an Inverted Phase Contrast Microscope to determine the estrous cycle stage through cytologic analysis. The procedure is done as described elsewhere ${ }^{10,11}$. Ensure the cell suspension does not dry out before cytologic analysis can be performed. The suspension may be diluted with fresh DPBS, if necessary.

\section{Mouse Reproductive Tract Ddissection}

1. Arrange the mouse in a supine position and spread its extremities to expose the abdominopelvic region.

2. Spray with $70 \%$ ethanol to moisten and disinfect the abdominopelvic area.

3. Using forceps, carefully lift the skin located superior to the clitoris. Make small transverse incisions on the lateral aspects of the lower abdominal area, up to the upper extremities to expose the peritoneum (Figure 1A). During this process, an external flap will form. As small incisions are continually made, the flap will increase in size.

4. Carefully cut through the peritoneum to expose the gastrointestinal tract (Figure 1B). It is important to note that the uterine horns can oftentimes be located directly under the peritoneum, so make incisions cautiously and do not touch the horns as this can affect uterine motility. 
5. Using forceps, remove the fascia and adipose tissue covering the gastrointestinal tract. Remove the following gastrointestinal tract segments from the abdominal cavity: the duodenum, jejunum, ileum, cecum, ascending and transverse colon (Figure 1C).

6. To locate the reproductive organs, first identify the urinary bladder (Figure 1C, "4"), which may have a deflated appearance due to voiding after euthanasia. The vagina will be right under the urinary bladder.

7. Locate the pubic symphysis at the confluence of the pubic bones (caudally to the bladder).

8. Using scissors, remove the pubic symphysis by carefully making incisions on its lateral sides through the interpubic fibrocartilaginous tissue to gain access to and provide a route for vagina extraction (Figure 1D).

9. Cut through the perineum located between the anus and lower part of the vulva.

10. Using forceps, lift the vagina and slowly excise the rectum.

11. Identify two uterine horns that bifurcate into a fork, rostrally to the vagina. Locate a convoluted oviduct and ovary at the end of each horn, which may be hidden under the remaining gastrointestinal tract segments. Use small dissecting scissors to remove any ligaments that connect to and support the horns, oviducts, and ovaries within the abdominal cavity.

12. Remove the reproductive tract, which includes the vagina, uterus, oviducts, and ovaries, from the abdominal cavity.

13. Transfer the isolated reproductive tract (Figure 1E) into a $100 \mathrm{~mm}$ Petri dish filled with $10 \mathrm{~mL}$ of DPBS. Do not compress the uterine horns to avoid damaging the myometrium.

14. Use forceps and surgical scissors to remove any connective and adipose tissue surrounding the uterine horns and vagina as well as any fur in the pubic region that could hinder the image quality. Remove the broad ligament to allow motility of the uterine horns.

15. Wash the isolated reproductive tract with fresh DPBS two times and transfer it into a $35 \mathrm{~mm}$ Petri dish filled with $3 \mathrm{~mL}$ of oxygenated Krebs solution.

\section{Tissue Imaging}

1. Place the Petri dish containing the reproductive tract in oxygenated Krebs buffer on a black surface. Maintain the dish at room temperature. NOTE: It is possible to use an infrared warming pad to maintain the tissue at $37^{\circ} \mathrm{C}$.

2. Allow $15-30$ min for spontaneous contractions to begin. Record spontaneous uterine motility for 10 min from an axial plane using any type of digital video equipment.

3. Transfer the preparation into a Petri dish containing the oxygenated Krebs buffer supplemented with a test compound. Record spontaneous uterine motility for about $10 \mathrm{~min}$ from an axial plane using any type of digital video equipment.

4. Wash the entire reproductive tract in $100 \mathrm{~mm}$ Petri dish with $10 \mathrm{~mL}$ of DPBS to assess the reversibility of treatment.

5. Transfer the preparation into a Petri dish with the freshly oxygenated Krebs buffer. Record spontaneous uterine motility for about 10 min from an axial plane using any type of digital video equipment.

6. Transfer the preparation to another $35 \mathrm{~mm}$ Petri dish filled with oxygenated Krebs buffer supplemented with the vehicle for the test compound to ensure that there are no mechanically induced changes in spontaneous uterine motility. This is an important control.

7. Transfer the video footage to a computer hard drive.

\section{Data Analysis}

1. Make clips using any video editing software from the original video footage containing the control, treatment, and wash episodes.

2. Use the MATLAB software and the provided script (see online Supplemental material) to quantify spontaneous uterine motility.

NOTE: Computer Vision Toolbox add-on for MATLAB must be installed for the script to be fully functional.

1. Open the MATLAB script, go to the Editor tab, and click Run.

2. Select the first video file and click Open.

3. Enter a label for the video file in the pop-up dialog box and click OK

4. Enter the time interval (s) needed for calculating the rate of horn movement ( $\Delta$ Euclidean distance / $\Delta \mathrm{s}$ ).

5. Use the mouse cursor to select two points on the first frame of the video. A pop-up window will ask to confirm that the selected points should be used for tracking. Click Start to initiate the real time tracking process that will be displayed in the pop-up window. Alternatively, click Reselect Points to reselect the two points.

6. Monitor the accuracy of the tracking process on the pop-up window.

7. Observe a rate vs. time scatter plot and distance vs. time scatter plot in a new pop-up window. Save the two figures by selecting File | Save As in the same window to document the data.

8. Find a folder named PointTrackerData, automatically created by MATLAB, in the same directory where the MATLAB script is located. Identify an Excel file named label_Data containing data points collected from the video in two separate worksheet tabs. NOTE: Any alternative motion tracking software can be used to quantify spontaneous uterus motility.

3. Use an appropriate software (e.g., Excel or SigmaPlot 13) to perform the statistical analysis.

\section{Representative Results}

Figure 1 shows representative images taken during the entire reproductive tract isolation procedure that is described in this protocol. To avoid contaminating the buffer with fur, which would decrease video quality, we moistened the mouse body with $70 \%$ ethanol. The major benchmark for the dissection section of the protocol is to find the urinary bladder. The uterus and vagina will be located inferior to the urinary bladder.

To test the protocol, we treated the entire reproductive tract with epinephrine. Epinephrine is well-known to cause uterine smooth muscle relaxation. This hormone is endogenously produced in the adrenal medulla and serves as a stress hormone in mammals. We used $1 \mu \mathrm{M}$ epinephrine in our experiments. This is a saturating concentration known to cause maximal response ${ }^{12}$. A series of four experiments were performed. In all trials, $1 \mu \mathrm{M}$ epinephrine reversibly inhibited spontaneously uterine motility (Figure 2). 
To quantify the spontaneous motility of the reproductive tract, we designed an algorithm allowing us to assess the average rate of change in the Euclidean distance between two selected points on the mouse reproductive tract. The point positions are tracked using the motion tracking module of MATLAB software. The corresponding script for MATLAB, which we used to calculate the Euclidean distances, is provided in the Supplemental material online. The position of points is critical for a successful motion tracking procedure. Careful consideration should be taken concerning the quality of the videos because the light reflections from the Petri dish's wall may distract the motion tracker, and it may stop tracking the horn movement while re-assigning the point to one of the light reflections. We opted to place one of the points in the middle of a horn to ensure that it was far enough from the Petri dish wall reflections. The second point was usually selected on the vagina since it did not exhibit spontaneous motility. Figure 3 provides a sample of data analysis, and Supplemental figure 1 shows the representative images acquired during motion tracking.

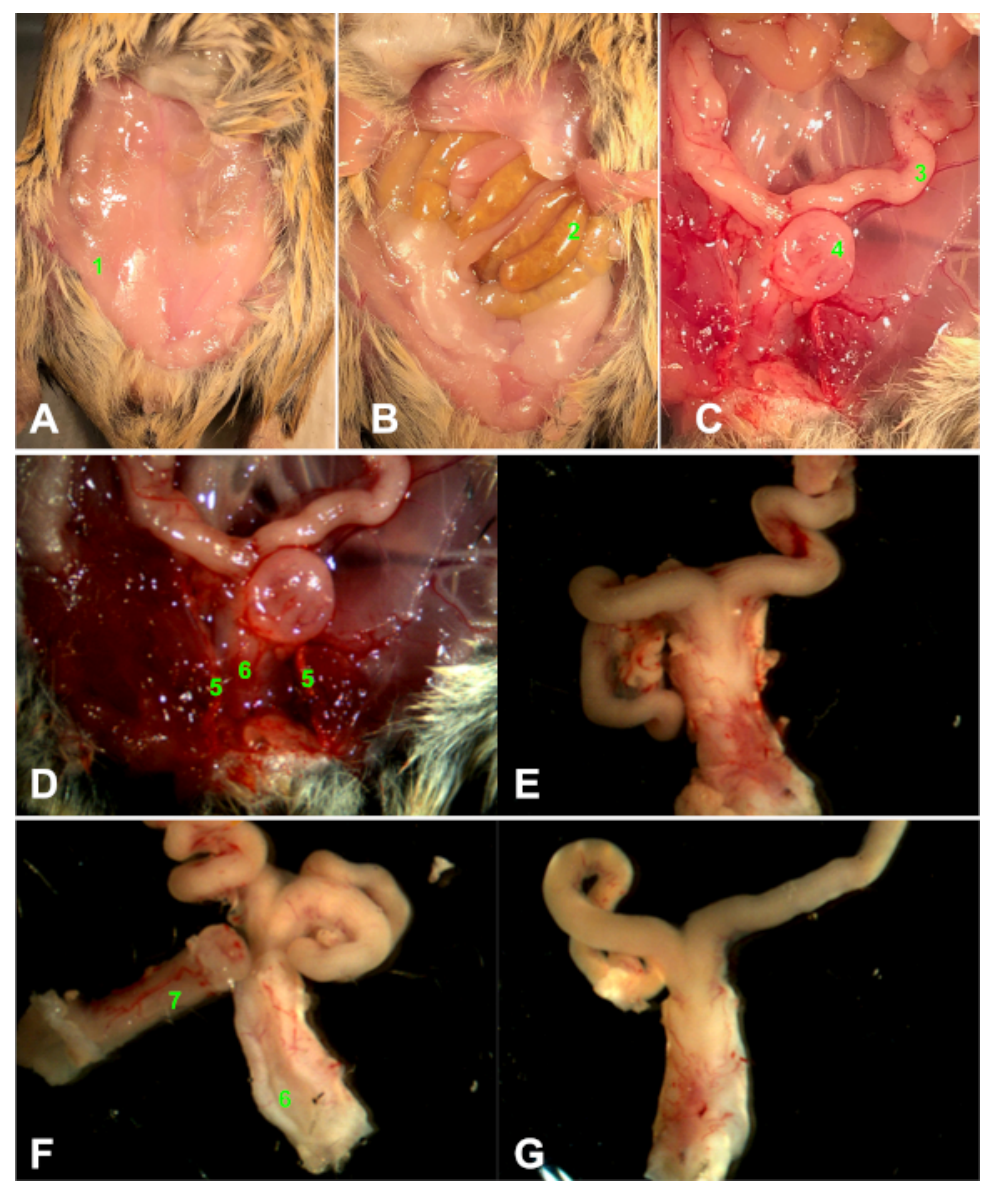

Figure 1: Steps of entire reproductive tract isolation. (A) An incision in the skin was made and the abdominopelvic region was exposed above the peritoneum (1). (B) The serous membrane was slowly opened to expose the gastrointestinal tract (2). (C) The gastrointestinal tract has been moved to expose the uterine horns (3). The urinary bladder (4) can be visualized near the conjunction of the horns. (D) The uterine horns have been freed and cuts have been made on the lateral sides of the pubic symphysis (5) to expose the vagina (6). (E) Removal of the isolated reproductive tract and placement into DPBS solution. Any excess fur or connective tissue was removed. $(\mathbf{F})$ A deep indentation can be seen on the vagina (right) after removal of the rectum (left, 7). (G) The surrounding connective tissue is removed. A digital camera and Application Suite software (version 3.7.0) was used to acquire real-time images during dissection (camera setting: hue 20/saturation 80). Please click here to view a larger version of this figure. 

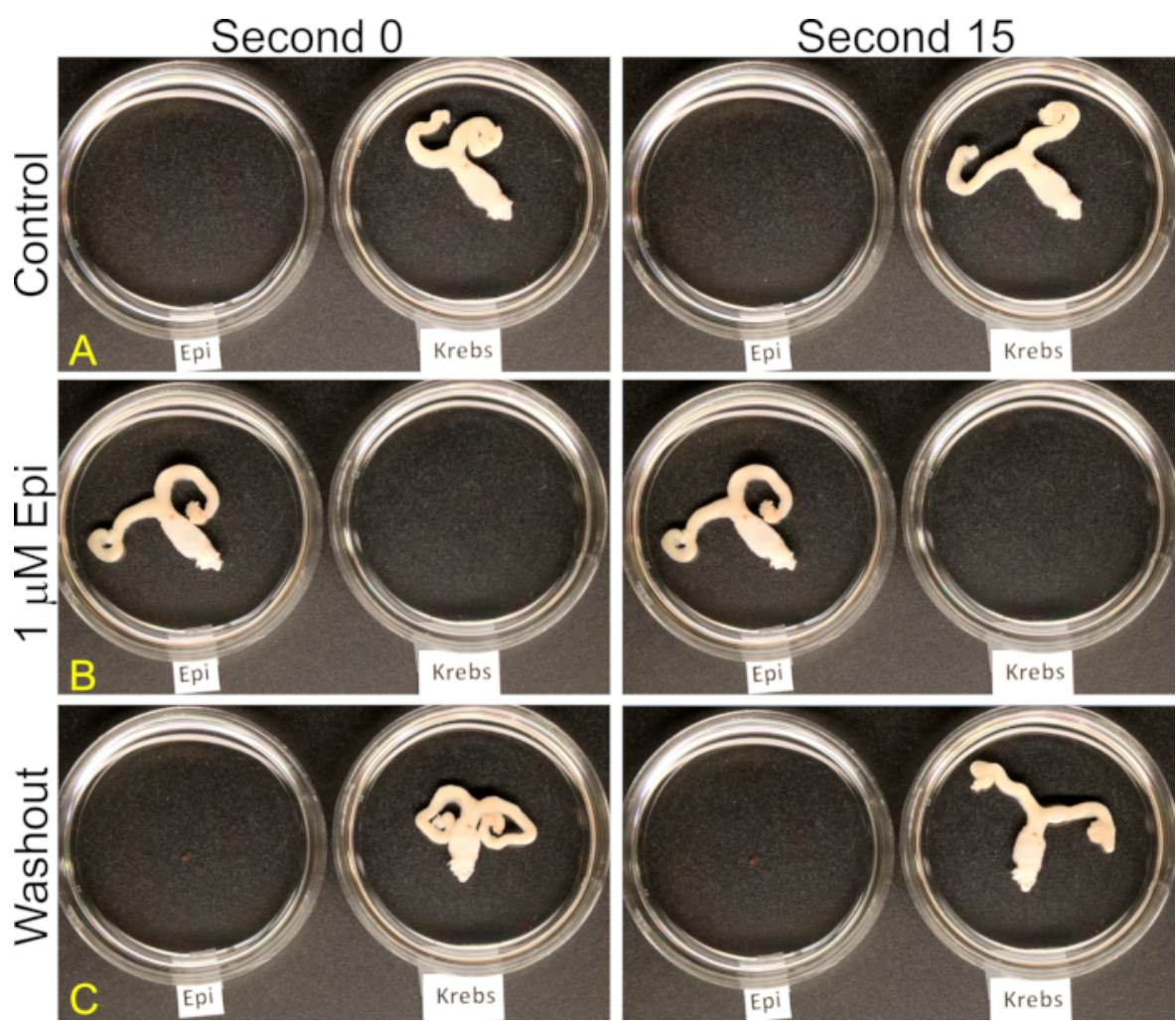

Figure 2: A representative experiment with the entire isolated reproductive tract is shown. The images were taken $15 \mathrm{~s}$ apart before (A), during (B), and after (C) the application of $1 \mu \mathrm{M}$ epinephrine. The reproductive tract preparation exhibited high motility in panels $\mathrm{A}$ and $\mathrm{C}$ in the absence of epinephrine, but it is quiescent in panel $B$ with the presence of $1 \mu \mathrm{M}$ epinephrine. The unedited video footage is provided as Supplemental videos 1-3. Please click here to view a larger version of this figure.
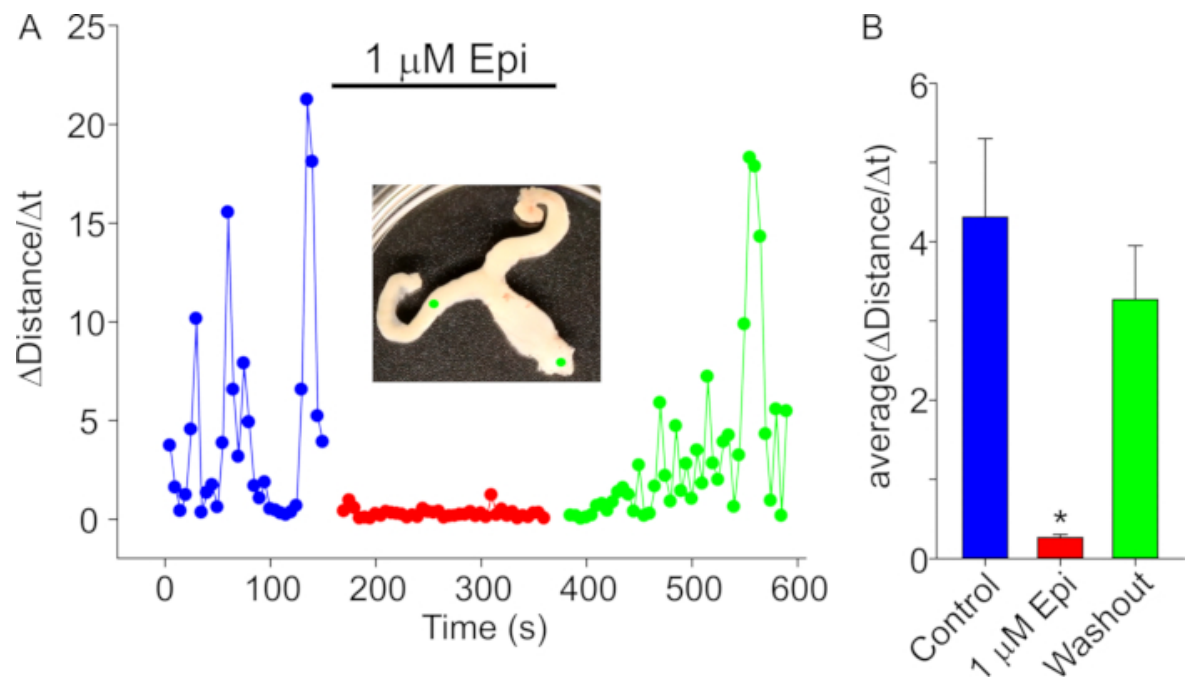

Figure 3: Data analysis in the ex vivo experiment described in Figure 2. (A) A time course of the Euclidean distance change rate is shown. The reference points between which the distance was determined during spontaneous uterine motility are shown as green dots in the inset. The points were selected at the proximal part of the vagina and the middle segment of a uterine horn as depicted. The blue filled circles show the spontaneous motility rate values before adding epinephrine, the red circles show the spontaneous motility rates in the presence of $1 \mu \mathrm{M}$ epinephrine, and the green filled circles show the spontaneous motility rates after a washout. (B) A comparison of average Euclidean distance change rates (pixels/s) before addition of epinephrine (blue bar), in the presence of $1 \mu \mathrm{M}$ epinephrine (red bar), and after a washout (green bar). The MATLAB software was used to quantify the uterine motility. $\Delta$ t interval was set at $5 \mathrm{~s}$. "DDistance" is calculated as the difference between the initial frame distance and the frame distance $5 \mathrm{~s}$ later. The statistical analysis was performed using Kruskal-Wallis One Way Analysis of Variance on Ranks followed by all pairwise multiple comparison procedures according to the Dunn's Method using SigmaPlot 13 . The asterisk indicates the data set that was significantly different from the other experimental data sets $(P=<0.001)$. Please click here to view a larger version of this figure. 


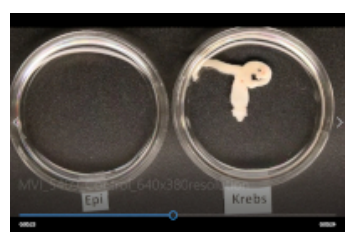

Supplemental movie 1: Time-lapse video clip showing spontaneous uterine motility before adding $1 \mu \mathrm{M}$ epinephrine. Please click here to view this video. (Right-click to download.)

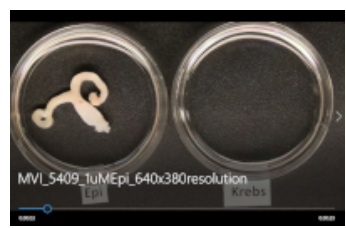

Supplemental movie 2: Time-lapse video clip showing spontaneous uterine motility when the Krebs buffer was supplemented with 1 $\mu \mathrm{M}$ epinephrine. Please click here to view this video. (Right-click to download.)

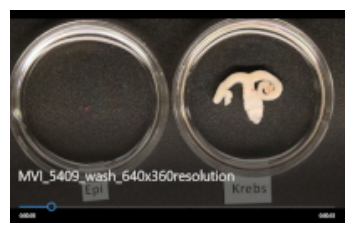

Supplemental movie 3: Time-lapse video clip showing spontaneous uterine motility after washout. Please click here to view this video. (Right-click to download.)

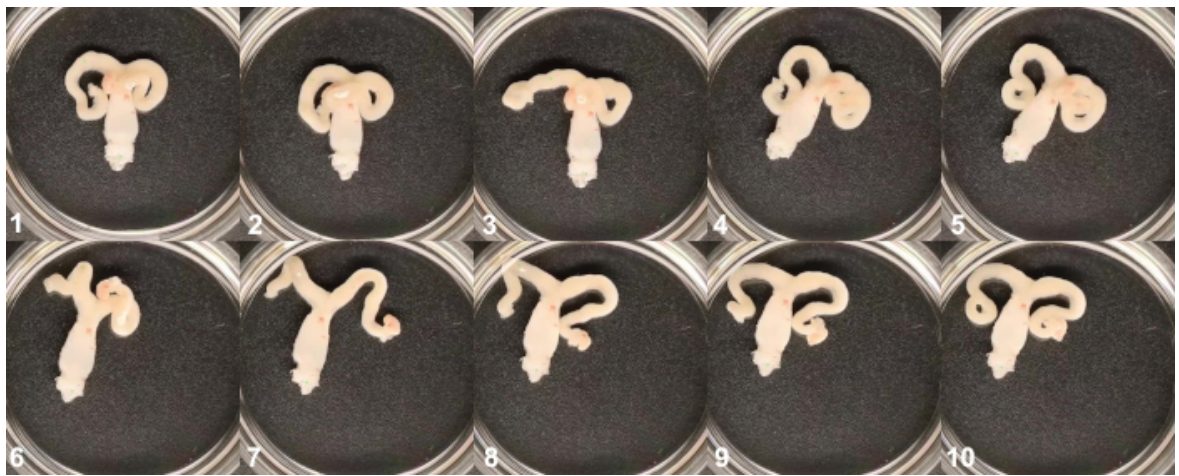

Supplemental Figure 1: Representative images taken every $15 \mathrm{~s}$ during motion tracking. Please click here to view a larger version of this figure.

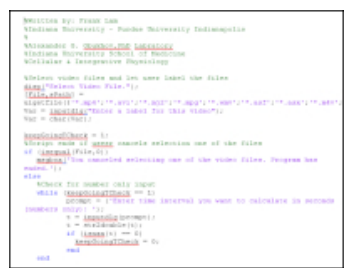

Supplemental Material: The MATLAB-based tracking algorithm script. Please click here to download this file.

\section{Discussion}

Here, we described a method for assessing spontaneous contractility of the entire rodent reproductive tract, which includes the ovaries, oviducts, uterine horns, and the vagina. We used a similar method to demonstrate the relaxant effect of phenylephrine on spontaneous uterine motility ${ }^{13}$, however, in the past we were unable to provide quantitative analysis of the data. In this work, we developed an algorithm for quantitative motility data analysis using the MATLAB motion tracking module. This is a useful technique for testing new drugs that regulate uterine contractility regardless of whether the drugs relax or constrict uterine smooth muscles. A major advantage of this model is that the reproductive tract is intact, preserving all intrinsic intrauterine cellular interactions. Notably, this protocol does not require any special equipment. The uterus isolation can be done with a simple magnifying glass and there is no requirement for any sophisticated video recording equipment. If a high-resolution digital camera is not available for imaging, a personal mobile phone camera may be used as an alternative.

The most critical step of the protocol for assessing reproductive tract motility is to obtain viable uterine tissue. The myometrium, within the uterine horns, is the motile element of the reproductive tract. Therefore, during isolation it is important to avoid overstretching or compressing the horns. 
It is also important to ensure that the uterine tissue is well-oxygenated throughout the experiment to maintain uterine motility. The best way to prevent any damage to the uterine horns is to make contact with only the adjacent connective tissue while cleaning the uterus or moving the reproductive tract. Since the vagina does not contract spontaneously during the experiment, it is acceptable to compress it with the forceps when moving the reproductive tract from one dish to another. The entire reproductive tract experiments may be performed in conjunction with isometric tension recordings that assess preload- and/or oxytocin-induced uterine contractility. However, a wire myograph is an expensive piece of equipment that is not always available in a common laboratory environment.

The described method has several limitations. Since the myometrium is very sensitive to compressions or pulls, this complicates the dissection process of the reproductive tract. If the horns are damaged during dissection, no spontaneous contractility will be observed. This is a major limitation of the protocol because it is uncertain whether the contractile smooth muscle cells were unknowingly damaged despite the use of proper care and caution or whether they lacked motility due to a natural cause. Indeed, we observed no motility in $10-20 \%$ of the reproductive tract preparations in this study. It is important to ensure that the vaginal segment of the reproductive tract remains intact because removal of the vagina markedly decreases spontaneous motility of the uterine horns. In contrast, the absence of ovaries and/or oviducts does not impair the entire reproductive tract motility. In addition, one should note that some compounds are sensitive to oxidation. For example, epinephrine can be easily oxidized. Testing the effects of such compounds would require shorter incubation time preventing excessive oxidation. However, shorter incubation times may hinder the ability of a drug to efficiently penetrate the thickness of the uterine wall. A further limitation of the method includes assessing the 3-dimensional movements of the horns. The horns have an innate nature of curling into a 3-dimensional plane, complicating analysis. To overcome this problem, one may decrease the Krebs buffer volume in the Petri dish to $2 \mathrm{~mL}$.

We found that the optimum age range for female mice is $2-5$ months. We indicated that anesthesia with isoflurane may result in reduced motility and additional washes may be necessary to prevent this isoflurane-induced complication. Alternatively, one can use carbon dioxide to euthanize mice. If difficulties arise during dissection, focus on landmarks, such as the urinary bladder, may help. To quantify the obtained motility data, we used MATLAB software. The major problem with motion tracking in MATLAB was that the tracker was unable to properly locate the horns when they moved near the wall of the Petri dish. Adobe Premier Element was used to clip the video footage and to reduce the size of video files. Although it is an outstanding software package, it may not always be available in a typical laboratory setting. An alternative option may be to use the free motion tracking module of Image $J$ software or even simple manual assessment of images taken with identical intervals and comparing the position of the uterine horns on a segmentation grid. Figure 2 shows an example of simple assessment of the spontaneous uterine motility and includes a comparison of reproductive tract movements within a $15 \mathrm{~s}$ interval.

No simple method assessing uterine motility in a Petri dish setting has been reported thus far. Ultrasound and intrauterine pressure sensors can be used to monitor human uterus contractility ${ }^{14}$. However, it is difficult to study underlying mechanisms in the intact human uterus. Therefore, the use of animal models for investigating spontaneous uterine contractility is important. The female reproductive tract exhibits spontaneous myometrial contractions that are critical for a woman's well-being, including her fertility and labor ${ }^{15}$. These contractions can be visualized as endometrial waves during an ultrasound examination. However, during menstruation, over contractility of the uterus can create discomfort and lead to dysmenorrhea, or menstrual cramps. To help relieve some symptoms of dysmenorrhea, new drugs targeted at relaxing uterine smooth muscles are needed. Our simple method provides a way for assessing the effects of various compounds on uterine contractility.

In this study, we used our easy Petri dish model to confirm the effectiveness of epinephrine, a uterine relaxant hormone ${ }^{12}$, to prevent spontaneous uterine motility of isolated mouse reproductive tracts. Our method may also be used for testing such compounds that may increase the contractility of the uterus. Importantly, this method might have the potential to be upgraded for throughput drug screening using a six-well plate format. Thus, the method we present here may have the capability to be optimized for industrial scale screening procedures. We did not attempt to perform similar experiments in larger rodents, but we expect that similar spontaneous motility will be present in isolated rat uteri. The larger reproductive tract of rats may exhibit more pronounced uterine motility. We found that pregnant mouse uteri may also be assessed using this ex vivo uterine motility Petri dish model. As expected, the spontaneous motility of the pregnant uterus was reduced because it is in a quiescent state. However, increased weight added by the fetal tissue may also contribute to motility obstruction. It may still be beneficial to further explore the suitability of this entire reproductive tract model to assess the effect of tocolytic (relaxant) or uterotonic (stimulant) compounds in the context of pregnancy and labor. Thus, here we presented an easy ex vivo model for assessing the spontaneous motility of the intact reproductive tract. This approach may be adopted for drug screening and be utilized for novel drug discovery.

\section{Disclosures}

The authors have nothing to disclose.

\section{Acknowledgments}

This work was supported by internal IU funds. AGO conceived the study. XC and AGO were involved in the design of the described experiments. FL and $A G O$ analyzed and interpreted the data. KLL, JOB, FL performed all of the ex vivo experiments. FL wrote the MATLAB script. KLL, JOB, and AGO wrote the manuscript. All authors read and approved the final version of the manuscript.

\section{References}

1. Kuijsters,N.P.M., et al. Uterine peristalsis and fertility: current knowledge and future perspectives: a review and meta-analysis. Reproductive BioMedicine Online. 35 (1), 50-71, (2017).

2. Kural,M., Noor,N.N., Pandit,D., Joshi,T., \& Patil,A. Menstrual characteristics and prevalence of dysmenorrhea in college going girls. Journal of Family Medicine and Primary Care. 4 (3), 426-431, (2015).

3. Dehnavi,Z.M., Jafarnejad,F., \& Kamali,Z. The Effect of aerobic exercise on primary dysmenorrhea: A clinical trial study. Journal of education and health promotion. 7, 3, (2018). 
4. Lindner,H.R., et al. Significance of prostaglandins in the regulation of cyclic events in the ovary and uterus. Advances in prostaglandin and thromboxane research. 8, 1371-1390 (1980).

5. Bernardi,M., Lazzeri,L., Perelli,F., Reis,F.M., \& Petraglia,F. Dysmenorrhea and related disorders. F1000 research. 6, 1645, (2017).

6. Marjoribanks,J., Ayeleke,R.O., Farquhar,C., \& Proctor,M. Nonsteroidal anti-inflammatory drugs for dysmenorrhoea. The Cochrane database of systematic reviews. 7, CD001751, (2015).

7. Oladosu,F.A., et al. Abdominal skeletal muscle activity precedes spontaneous menstrual cramping pain in primary dysmenorrhea. American journal of obstetrics and gynecology. 219 (1), 91, (2018).

8. Lawson,C.C., et al. Occupational exposures among nurses and risk of spontaneous abortion. American journal of obstetrics and gynecology. 206 (4), 327-328, (2012).

9. Mazze,R.I., Wilson,A.I., Rice,S.A., \& Baden,J.M. Fetal development in mice exposed to isoflurane. Teratology. 32 (3), 339-345, (1985).

10. Byers,S.L., Wiles,M.V., Dunn,S.L., \& Taft,R.A. Mouse estrous cycle identification tool and images. PLoS One. 7 (4), e35538, (2012).

11. Caligioni,C.S. Assessing reproductive status/stages in mice. Current protocols in neuroscience. Appendix 4, Appendix, (2009).

12. Segal,S., Csavoy,A.N., \& Datta,S. The tocolytic effect of catecholamines in the gravid rat uterus. Anesthesia and analgesia. 87 (4), $864-869$ (1998).

13. Chen,X., et al. Phenylephrine, a common cold remedy active ingredient, suppresses uterine contractions through cAMP signalling. Scientific reports. 8 (1), 11666, (2018)

14. Domino,M., Pawlinski,B., \& Gajewski,Z. Biomathematical pattern of EMG signal propagation in smooth muscle of the non-pregnant porcine uterus. PLoS One. 12 (3), e0173452, (2017).

15. Crane,L.H., \& Martin,L. Pace-maker activity in the myometrium of the oestrous rat: in vivo studies using video-laparoscopy. Reproduction, fertility, and development. 3 (5), 519-527 (1991). 